\title{
Artificially disordered birefringent optical fibers
}

\author{
S. Herath, ${ }^{1}$ N. P. Puente, ${ }^{2}$ E. I. Chaikina, ${ }^{3}$ and A. Yamilov ${ }^{1, *}$ \\ ${ }^{1}$ Department of Physics, Missouri University of Science \& Technology, Rolla, Missouri 65409, \\ USA \\ ${ }^{2}$ Universidad Autónoma de Nuevo León, UANL, FIME, Av. Universidad S/N Ciudad \\ Universitaria San Nicolás de los Garza Nuevo León, C.P. 66451, México \\ ${ }^{3}$ División de Física Aplicada, Centro de Investigación Científica y de Educación Superior de \\ Ensenada,B.C., 22860, Ensenada, México \\ *yamilov@mst.edu
}

\begin{abstract}
We develop and experimentally verify a theory of evolution of polarization in artificially-disordered multi-mode optical fibers. Starting with a microscopic model of photo-induced index change, we obtain the first and second order statistics of the dielectric tensor in a Ge-doped fiber, where a volume disorder is intentionally inscribed via UV radiation transmitted through a diffuser. A hybrid coupled-power \& coupled-mode theory is developed to describe the transient process of de-polarization of light launched into such a fiber. After certain characteristic distance, the power is predicted to be equally distributed over all co-propagating modes of the fiber regardless of their polarization. Polarization-resolved experiments, confirm the predicted evolution of the state of polarization. Complete mode mixing in a segment of fiber as short as $\sim 10 \mathrm{~cm}$ after $3.6 \mathrm{~dB}$ insertion loss is experimentally observed. Equal excitation of all modes in such a multi-mode fiber creates the conditions to maximize the information capacity of the system under e.g. multiple-input-multiple-output (MIMO) transmission setup.
\end{abstract}

(C) 2012 Optical Society of America

OCIS codes: (290.4210) Multiple scattering; (060.2310) Fiber optics; (260.1440) Birefringence; (290.5825) Scattering theory.

\section{References and links}

1. A. Ishimaru, Wave Propagation and Scattering in Random Media (Academic Press, 1978).

2. A. F. Garito, J. Wang, and R. Gao, "Effects of random perturbations in plastic optical fibers," Science 281, 962 967 (1998).

3. H. Cao, "Lasing in disordered media," in Progress in Optics, E. Wolf, ed. (North Holland, 2003), Vol. 45.

4. S. E. Skipetrov, "Disorder is the new order," Nature 432, 285-286 (2004).

5. I. M. Vellekoop, A. Lagendijk, and A. P. Mosk, "Exploiting disorder for perfect focusing," Nat. Photonics 4, 320-322 (2010).

6. M. Limonov and R. D. L. Rue, eds., Optical Properties of Photonic Structures: Interplay of Order and Disorder (Francis \& Taylor, 2012).

7. M. Fink, "Time reversed acoustics," Phys. Today 50, 34-40 (1997).

8. X. Shen, J. M. Kahn, and M. A. Horowitz, "Compensation for multimode fiber dispersion by adaptive optics," Opt. Lett. 30, 2985-2987 (2005).

9. A. Amphawan, "Holographic mode-selective launch for bandwidth enhancement in multimode fiber," Opt. Express 19, 9056-9065 (2011).

10. G. J. Foschini and M. J. Gans, "On limits of wireless communications in a fading environment when using multiple antennas," Wireless Personal Commun. 6, 311-335 (1998). 
11. E. Telatar, "Capacity of multi-antenna gaussian channels," Euro. Trans. Telecommun. 10, 585-595 (1999).

12. S. H. Simon, A. L. Moustakas, M. Stoychev, and H. Safar, "Communication in a disordered world," Phys. Today 54, 38-43 (2001)

13. H. R. Stuart, "Dispersive multiplexing in multimode optical fiber," Science 289, 281-283 (2000).

14. M. Nazarathy and A. Agmon, "Coherent transmission direct detection MIMO over short-range optical interconnects and passive optical networks," J. Lightwave Technol. 26, 2037-2045 (2008).

15. M. Greenberg, M. Nazarathy, and M. Orenstein, "Multimode fiber as random code generator-application to massively parallel MIMO transmission," J. Lightwave Technol. 26, 882-890 (2008).

16. R. C. J. Hsu, A. Tarighat, A. Shah, A. H. Sayed, and B. Jalali, "Capacity enhancement in coherent optical MIMO (COMIMO) multimode fiber links," IEEE Commun. Lett. 10, 195-197 (2006).

17. N. P. Puente, E. I. Chaikina, S. Herath, and A. Yamilov, "Fabrication, characterization, and theoretical analysis of controlled disorder in the core of optical fibers," Appl. Opt. 50, 802-810 (2011).

18. D. Marcuse, Theory of Dielectric Optical Waveguides (Academic, 1974).

19. D. Gloge, "Optical power flow in multimode fibers," Bell Syst. Tech. J. 51, 1767-1783 (1972).

20. R. Olshansky, "Mode coupling effects in graded-index optical fibers," Appl. Opt. 14, 935-945 (1975).

21. K. O. Hill and G. Meltz, "Fiber Bragg grating technology: Fundamentals and overview," J. Lightwave Technol. 15, 1263-1276 (1997).

22. T. Erdogan, "Fiber grating spectra," J. Lightwave Technol. 15, 1277 -1294 (1997).

23. A. Kamal and P. S. J. Russell, "Physical origins and general dielectric tensor of photoinduced anisotropy in optical fibers and bulk glasses," J. Opt. Soc. Am. B 11, 1576-1584 (1994).

24. T. Erdogan and V. Mizrahi, "Characterization of UV-induced birefringence in photosensitive Ge-doped silica optical fibers," J. Opt. Soc. Am. B 11, 2100-2105 (1994).

25. A. M. Vengsarkar, Q. Zhong, D. Inniss, W. A. Reed, P. J. Lemaire, and S. G. Kosinski, "Birefringence reduction in side-written photoinduced fiber devices by a dual-exposure method," Opt. Lett. 19, 1260-1262 (1994).

26. N. Belhadj, Y. Park, S. LaRochelle, K. Dossou, and J. Azana, "UV-induced modification of stress distribution in optical fibers and its contribution to bragg grating birefringence," Opt. Express 16, 8727-8741 (2008).

27. R. K. Luneburg, Mathematical Theory of Optics (Univ. California Press, 1964).

28. J. W. Goodman, Speckle Phenomena in Optics: Theory and Applications (Coberts \& Co, Englewood, 2007).

29. G. J. Foschini and C. D. Poole, "Statistical theory of polarization dispersion in single mode fibers," J. Lightwave Technol. 9, 1439-1456 (1991).

30. J. P. Gordon and H. Kogelnik, "PMD fundamentals: Polarization mode dispersion in optical fibers," Proc. Natl. Acad. Sci. USA 97, 4541-4550 (2000).

31. D. Marcuse, "Coupled power equations for lossy fibers," Appl. Opt. 17, 3232-3237 (1978).

32. L. Mandel and E. Wolf, Optical Coherence and Quantum Optics (Cambridge University Press, New York, 1995).

33. O. Korotkova, "Changes in statistics of the instantaneous stokes parameters of a quasi-monochromatic electromagnetic beam on propagation,” Opt. Commun. 261, 218-224 (2006).

\section{Introduction}

The last decade has witnessed a shift in the general perception of disorder in optical systems from a nuisance [1] to an exploitable feature which may enable some unique functionalities [2-6]. An ability to control light propagation in complex media [5] via spatial light modulator (SLM) exposed a deep connection to the problem of time reversal in acoustics [7] and renewed interest in such systems. SLM has also been used to manipulate certain aspects of propagation, e.g. to compensate modal dispersion, in multi-mode optical fibers MMF $[8,9]$.

Mutiple-input-multiple-output (MIMO) approach [10-12] has become the backbone of the wireless IEEE 802.11n standard for local area networks (LANs). It takes advantage of the differences in propagation from multiple sources to multiple receivers to maximize the bandwidth of the transmitted signal - with the theoretical limit of improvement over single-input-singleoutput (SISO) being proportional to number of sources or receivers, whichever is smallest [11].

A similar approach has also been applied to MMF [13]. It relies on the modal-coupling diversity (MCD) to take advantage of all available degrees of freedom in a MMF. Importantly, the method does not require orthogonal coupling to the individual modes of the fiber and its effectiveness is not degraded by inter-mode coupling in the fiber $[14,15]$. To maximize MCD, the mode coupling coefficients in the fiber link should be sufficiently random - Gaussian [11, 16]. In conventional fibers, meeting this condition requires sufficiently long segments of MMF because concentration of imperfections is low. In the most disordered case of plastic optical 
fibers (POF) the characteristic length for mode mixing is on the order of $\sim 10 \mathrm{~m}$ [2]. Improving the efficiency of inter-mode coupling and randomization by increasing the concentration of defects has the drawback of enhanced losses.

In this work we investigate both theoretically and experimentally photo-sensitive multi-mode Ge-doped silica fibers with artificially induced disorder. Our thorough account of the photoinduced birefringence allows us to develop a theory which captures the effect of direct coupling between two sets of modes with both orthogonal polarizations. Experimental observations demonstrate that the transition to the fully mode-mixed state occurs in very short fibers of length $\sim 10 \mathrm{~cm}$. Because of such short length of the fiber, other sources of birefringence can be neglected. Furthermore, it should be possible to mitigate the trade-off between strength of intermode coupling and the radiative losses by controlling spatial correlations of the disorder [17].

Coupled-power theory [18] has been widely used to describe the modal distribution, as well as the temporal characteristics of pulse propagation and pulse bandwidth $[2,19,20]$. However, power-coupling models fail to properly describe the effect of birefringence because the latter affects the amplitudes and phases of the cross-polarized modes. Here we develop a hybrid coupled-power/coupled-mode approach which shows that the limiting modal distribution is uniform - all co-propagating modes (including those with orthogonal polarization) of the MMF are statistically equally excited. This implies optimum MCD to maximize the information capacity in transmission through MMF. Moreover, the developed formalism allowed us to describe and interpret a crossover from an initial mode distribution to the limiting one.

\section{Properties of photo-induced disorder}

\subsection{Microscopic model of birefringence}

Photo-sensitivity of the germano-silicate fibers [21] allows one to modify the refractive index in the core of optical fibers. This effect has been used to laser-write a wide variety of fiber gratings [22]. Doping by germanium atoms leads to oxygen-deficient defect formation. Illumination by a UV source with a sufficiently short wavelength breaks these bonds that, in turn, modifies the absorption spectrum of the glass. The real part of the refractive index is changed through the Kramers-Kronig relation.

It has also been observed that under certain conditions the photo-modified fibers become birefringent. Three contributions to photo-induced birefringence have been identified: (i) a contribution which depends on the polarization of the UV laser source [23, 24]; (ii) a contribution due to spatial non-uniformity of the incident UV beam [25] ; and (iii) a contribution related to modification in glass stresses [26]. Commonly, the first contribution is the dominant one. A detailed microscopical model describing the dependence of the birefringence on the polarization state of the UV radiation was developed Kamal and Russell in Ref. [23].

The Kamal-Russell model relates the macroscopic change in the local susceptibility tensor $\Delta \chi_{i j}(\vec{r})$ to the change in the volume density of the highly directional breakable bonds $\Delta \rho(\vec{r}, \theta, \phi)$ in the germano-silicate glass as

$$
\Delta \chi_{i j}(\vec{r})=\alpha_{b} \int \Delta \rho(\vec{r}, \theta, \phi) u_{i} u_{j} d \Omega,
$$

where $\alpha_{b}$ is the polarizability of the bond, $\vec{u}=(\sin \theta \cos \phi, \sin \theta \sin \phi, \cos \theta)$ define the orientation of the bond, and the integral is taken of over all solid angles $\Omega$. The process of severing the bond is described by the Fermi golden rule with the dipole - electric field interaction Hamiltonian. The change $\Delta \rho(\vec{r}, \theta, \phi)$ in the initially isotropically uniform distribution $\rho /(4 \pi)$ is related to the cumulative effect of UV radiation during the time of the exposure. Photo-induced change 
in the susceptibility tensor is then related to the UV electric fields

$$
\begin{aligned}
\Delta \chi_{x x}(\vec{r}) & =C_{0}\left[3\left|E_{x}^{(U V)}(\vec{r})\right|^{2}+\left|E_{y}^{(U V)}(\vec{r})\right|^{2}+\left|E_{z}^{(U V)}(\vec{r})\right|^{2}\right] \\
\Delta \chi_{x y}(\vec{r}) & =C_{0}\left[E_{x}^{(U V)}(\vec{r}) E_{y}^{(U V) *}(\vec{r})+E_{x}^{(U V) *}(\vec{r}) E_{y}^{(U V)}(\vec{r})\right]
\end{aligned}
$$

where $C_{0}$ encompasses all material-related parameters, not essential to the further discussion. The remaining components of the tensor are obtained from Eq. (2) and Eq. (3) via permutation of the subscripts. Using this result, in the next section we obtain the relationship between statistics of the speckle pattern of the incident UV light and the spatial correlation of the elements of the dielectric tensor.

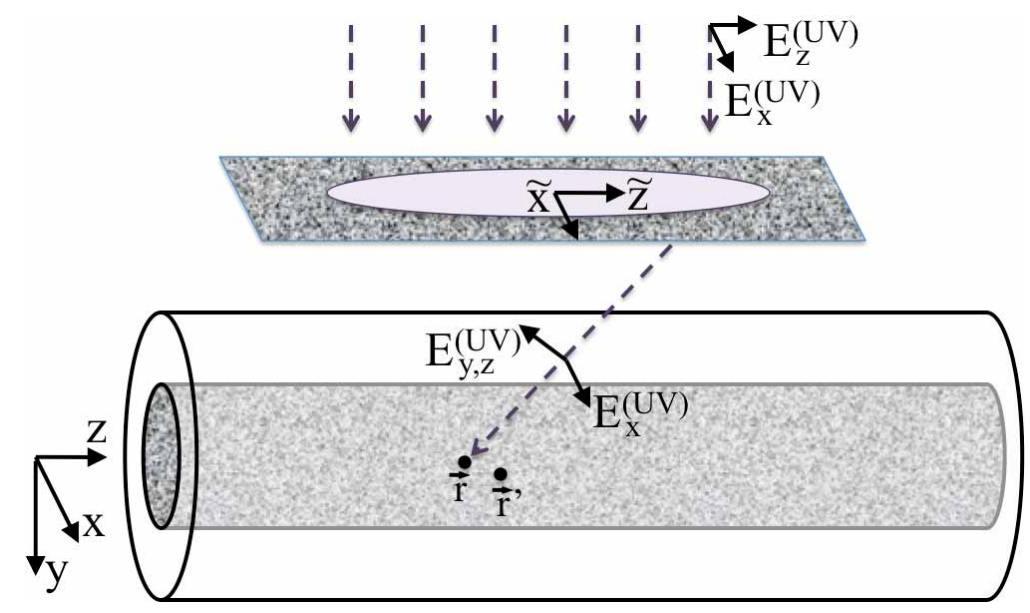

Fig. 1. UV irradiation geometry is shown. Expanded unpolarized $\lambda_{U V}=244 \mathrm{~nm}$ UV light from an Ar laser illuminates an elongated area on the surface of a diffuser. A complex interference pattern is incident onto the core of the Ge-doped photosensitive fiber.

\subsection{Statistical properties of the UV light used to fabricate the disorder}

As we will see in the following sections, to describe coupling between different modes of our disordered fiber we need first to determine the spatial correlations of the random fluctuations of the dielectric tensor around its average $n_{\text {core }}^{2}+\left\langle\Delta \chi_{i j}(\vec{r})\right\rangle$

$$
\left\langle\delta \varepsilon_{i j}(\vec{r}) \delta \varepsilon_{i j}\left(\vec{r}^{\prime}\right)\right\rangle \equiv\left\langle\left(\Delta \chi_{i j}(\vec{r})-\left\langle\Delta \chi_{i j}(\vec{r})\right\rangle\right) \cdot\left(\Delta \chi_{i j}\left(\vec{r}^{\prime}\right)-\left\langle\Delta \chi_{i j}\left(\vec{r}^{\prime}\right)\right\rangle\right)\right\rangle .
$$

Equation (2) and Eq. (3) relate the change of susceptibilities to the electric field used to fabricate the pattern. Hence the problem of obtaining correlators in Eq. (4) reduces to the problem in determining correlations between UV fields. Below, we proceed to determine the statistical properties of $E_{i}^{(U V)}(\vec{r})$.

Vector components of the UV electric field produced by the diffuser, c.f. Fig. 1, can be found with the help of Rayleigh-Sommerfeld vector diffraction theory [27]:

$$
\begin{aligned}
E_{x}^{(U V)}(\vec{r}) & =-\frac{D+y}{2 \pi} \iint \frac{i k \exp [i k R]}{R^{2}} E_{\tilde{x}}^{(U V)}(\tilde{x}, \tilde{z}) d \tilde{x} d \tilde{z} \\
E_{y}^{(U V)}(\vec{r}) & =\frac{1}{2 \pi} \iint \frac{i k \exp [i k R]}{R^{2}}\left(E_{\tilde{x}}^{(U V)}(\tilde{x}, \tilde{z})(x-\tilde{x})+E_{\tilde{z}}^{(U V)}(\tilde{x}, \tilde{z})(z-\tilde{z})\right) d \tilde{x} d \tilde{z},
\end{aligned}
$$


In these equations we adopt the following notations: $(\tilde{x}, \tilde{z})$ are coordinates in the plane of the diffuser; $\vec{r}=(x, y, z)$ are coordinates in the fiber core; $E_{\tilde{x}}^{(U V)}(\tilde{x}, \tilde{z})$ and $E_{\tilde{z}}^{(U V)}(\tilde{x}, \tilde{z})$ are two components of the UV electric fields in the plane of the diffuser; $E_{i}^{(U V)}(\vec{r})$ are the UV field components in the fiber core; $R$ denotes the distance from $(\tilde{x}, \tilde{z})$ to $\vec{r}$; and $k=2 \pi / \lambda_{U V}$. Expression for $E_{z}^{(U V)}(\vec{r})$ is analogous to Eq. (5).

We assume that the fields originating at the diffuser are $\delta$-correlated Gaussian [28]:

$$
\begin{array}{r}
\left\langle E_{\tilde{x}}^{(U V)}(\tilde{x}, \tilde{z}) E_{\tilde{x}}^{(U V) *}\left(\tilde{x}^{\prime}, \tilde{z}^{\prime}\right)\right\rangle=\left\langle E_{\tilde{z}}^{(U V)}(\tilde{x}, \tilde{z}) E_{\tilde{z}}^{(U V) *}\left(\tilde{x}^{\prime}, \tilde{z}^{\prime}\right)\right\rangle=(1 / 2) I_{0}^{(U V)}(\tilde{x}, \tilde{z}) \kappa \delta\left(\tilde{x}-\tilde{x}^{\prime}\right) \delta\left(\tilde{z}-\tilde{z}^{\prime}\right), \\
\left\langle E_{\tilde{x}}^{(U V)}(\tilde{x}, \tilde{z}) E_{\tilde{x}}^{(U V)}\left(\tilde{x}^{\prime}, \tilde{z}^{\prime}\right)\right\rangle=\left\langle E_{\tilde{z}}^{(U V)}(\tilde{x}, \tilde{z}) E_{\tilde{z}}^{(U V)}\left(\tilde{x}^{\prime}, \tilde{z}^{\prime}\right)\right\rangle=\left\langle E_{\tilde{x}}^{(U V)}(\tilde{x}, \tilde{z}) E_{\tilde{z}}^{(U V)}\left(\tilde{x}^{\prime}, \tilde{z}^{\prime}\right)\right\rangle=0
\end{array}
$$

where $I_{0}^{(U V)}(\tilde{x}, \tilde{z})=\left(\pi L_{x} L_{z}\right)^{-1} \times \exp \left[-\tilde{x}^{2} / L_{x}^{2}-\tilde{z}^{2} / L_{z}^{2}\right]$ is a Gaussian beam profile, expanded along $z$-axis, which illuminates the diffuser. Using Eq. (5), Eq. (6), Eq. (7) and Eq. (8) we obtain

$$
\left\langle E_{x}^{(U V)}(\vec{r}) E_{x}^{(U V) *}\left(\vec{r}^{\prime}\right)\right\rangle=\frac{\kappa(D+y)\left(D+y^{\prime}\right)}{\lambda_{U V}^{2}} \iint \frac{\exp \left[i k\left(R-R^{\prime}\right)\right]}{R^{2} R^{\prime 2}} I_{0}^{(U V)}(\tilde{x}, \tilde{z}) d \tilde{x} d \tilde{z} .
$$

The correlation between the other components of the field can be obtained analogously. Because $E_{y}^{(U V)}(\vec{r})$ depends on the both $E_{\tilde{x}}^{(U V)}\left(\tilde{x}^{\prime}, \tilde{z}^{\prime}\right)$ and $E_{\tilde{z}}^{(U V)}\left(\tilde{x}^{\prime}, \tilde{z}^{\prime}\right)$ in Eq. (6), $\left\langle E_{x}^{(U V)}(\vec{r}) E_{y}^{(U V) *}\left(\vec{r}^{\prime}\right)\right\rangle$ and $\left\langle E_{y}^{(U V)}(\vec{r}) E_{z}^{(U V) *}\left(\vec{r}^{\prime}\right)\right\rangle$ remain non-zero.
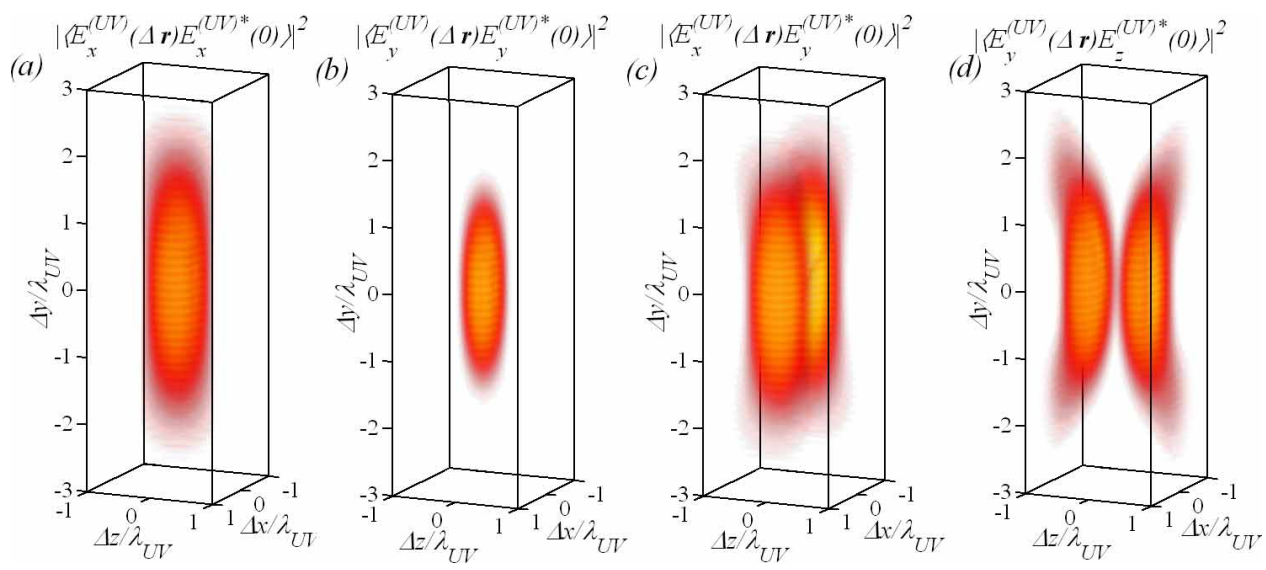

Fig. 2. Correlations between field components of the UV light used to fabricate disorder in the core of the photo-sensitive optical fiber. Equation (9) and similar expression for the other field components, originating from Rayleigh-Sommerfeld integrals in Eq. (5) and Eq. (6), are evaluated numerically under the experimentally relevant conditions $L_{x}=3 \mathrm{~mm}, L_{z}=5 \mathrm{~mm}, D=5 \mathrm{~mm}$. Not shown are $\left\langle E_{x}^{(U V)}(\vec{r}) E_{z}^{(U V) *}\left(\vec{r}^{\prime}\right)\right\rangle$ which vanishes completely and $\left\langle E_{z}^{(U V)}(\vec{r}) E_{z}^{(U V) *}\left(\vec{r}^{\prime}\right)\right\rangle$ which is identical to $\left\langle E_{x}^{(U V)}(\vec{r}) E_{x}^{(U V) *}\left(\vec{r}^{\prime}\right)\right\rangle$.

Analytical evaluation of Eq. (9) and the corresponding expressions for the other combinations of the field components proves to be challenging under the experimentally relevant conditions, c.f. Ref. [17]. Indeed, the mathematically convenient paraxial approximation is not well justified because the dimensions of the illuminated spot, $L_{x}$ and $L_{z}$, are comparable to the distance to fiber core $D$. Furthermore, under this approximation $E_{y}^{(U V)}(\vec{r})$ vanishes entirely and the effect

\#160441 - \$15.00 USD

(C) 2012 OSA
Received 22 Dec 2011; revised 11 Jan 2012; accepted 11 Jan 2012; published 30 Jan 2012 13 February 2012 / Vol. 20, No. 4 / OPTICS EXPRESS 3624 
of cross-polarization mode coupling is washed out even though the fiber becomes birefringent because of the strong $E_{x}^{(U V)}(\vec{r})$.

To circumvent the limitations imposed by the paraxial approximation in Eq. (9), we evaluated the integrals numerically. The results shown in Fig. 2 demonstrate that photoinduced speckle pattern is highly anisotropic. Moreover, correlators $\left\langle E_{x}^{(U V)}(\vec{r}) E_{y}^{(U V) *}\left(\vec{r}^{\prime}\right)\right\rangle$ and $\left\langle E_{y}^{(U V)}(\vec{r}) E_{z}^{(U V) *}\left(\vec{r}^{\prime}\right)\right\rangle$ field components have $y-z$ and $x-y$ nodal planes respectively due to symmetries in the integral in Eq. (6) defining $y$-component of the field. $\left\langle E_{x}^{(U V)}(\vec{r}) E_{z}^{(U V) *}\left(\vec{r}^{\prime}\right)\right\rangle$ vanishes completely and correlator $\left\langle E_{z}^{(U V)}(\vec{r}) E_{z}^{(U V) *}\left(\vec{r}^{\prime}\right)\right\rangle$ is identical to Eq. (9). We note that the effect of refraction of the incident UV light at the air-cladding interface is not accounted for in the derivation of Eq. (9), but it is not expected to change our results qualitatively. However we do have to scale the spatial dimensions of the speckles by the refractive index of the fiber core. This can be accomplished by $\lambda_{U V} \rightarrow \lambda_{U V} / n_{\text {core }}$ substitution in Fig. 2 and Fig. 3.

Scalar theory [17] yields zero $\left\langle\delta \varepsilon_{x y}(\vec{r}) \delta \varepsilon_{x y}\left(\vec{r}^{\prime}\right)\right\rangle$ and, hence, it does not contain a mechanism to explain cross-polarization coupling by the photo-induced disorder. However, the scalar theory correctly predicts the highly anisotropic structure of correlations in the diagonal components of the dielectric tensor $\left\langle\delta \varepsilon_{i i}(\vec{r}) \delta \varepsilon_{i i}\left(\vec{r}^{\prime}\right)\right\rangle$. This is a consequence of Eq. (2) and Eq. (4) (see also Fig. 2(a) and Fig. 2(b)) which is linked to suppression of the radiative leakage in such a fiber [17]. We expect the same conclusion to hold for theory presented in this work, which properly accounts for the birefringence effects.

Due to non-uniform illumination of the diffuser, the correlators $\left\langle E_{i}^{(U V)}(\vec{r}) E_{j}^{(U V) *}\left(\vec{r}^{\prime}\right)\right\rangle$ depend not only on $\Delta \vec{r} \equiv \vec{r}-\vec{r}^{\prime}$ but also on $\left(\vec{r}+\vec{r}^{\prime}\right) / 2$. However, we find that this dependence affects only weakly both magnitude and the shape of the field-field correlators for $-L_{z} / 2<\left(z+z^{\prime}\right) / 2<L_{z} / 2$.
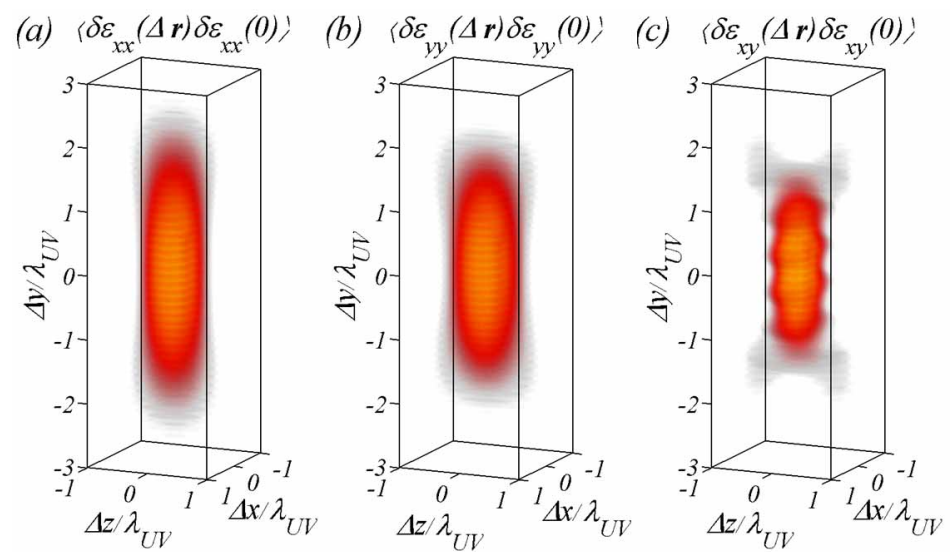

Fig. 3. Statistical properties of the spatially fluctuating dielectric tensor are described by correlators $\left\langle\delta \varepsilon_{i j}(\vec{r}) \delta \varepsilon_{i j}\left(\vec{r}^{\prime}\right)\right\rangle$ in Eqs. (12). To evaluate these expression we used the same set of parameters as in Fig. 2. Only the $x-y$ part of the entire tensor relevant to inter-mode coupling are shown.

\subsection{Statistical properties of disorder}

In the next step we obtain the expressions for the photo-induced changes in the dielectric tensor and its fluctuations. Assuming that individual field components represent Gaussian random 
variables we find the following expression for the change in the spatial average dielectric tensor $\langle\hat{\varepsilon}\rangle-n_{\text {core }}^{2}$ :

$$
\begin{aligned}
& \Delta \varepsilon_{x x}=\left\langle\varepsilon_{x x}\right\rangle-n_{\text {core }}^{2}=C_{0}\left(4\left\langle\left|E_{x}^{(U V)}\right|^{2}\right\rangle+2\left\langle\left|E_{y}^{(U V)}\right|^{2}\right\rangle\right) \\
& \Delta \varepsilon_{y y}=\left\langle\varepsilon_{y y}\right\rangle-n_{\text {core }}^{2}=C_{0}\left(2\left\langle\left|E_{x}^{(U V)}\right|^{2}\right\rangle+3\left\langle\left|E_{y}^{(U V)}\right|^{2}\right\rangle\right)
\end{aligned}
$$

where all off-diagonal elements are equal to zero, $\left\langle\varepsilon_{x x}\right\rangle=\left\langle\varepsilon_{z z}\right\rangle$, and we assumed that the diffuser in Fig. 1 is illuminated by an unpolarized beam, i.e. $\left\langle\left|E_{x}^{(U V)}\right|^{2}\right\rangle=\left\langle\left|E_{z}^{(U V)}\right|^{2}\right\rangle$. We immediately observe that the change is anisotropic leading to birefringence. This is an effect well known in fabrication of Bragg gratings [22]. The birefringence along the fiber axis can be suppressed by polarizing the UV light along the axis of the fiber ( $z$-axis), which is in accordance with experiments [24].

Statistical properties of the spatially fluctuating dielectric tensor are described by $\left\langle\delta \varepsilon_{i j}(\vec{r}) \delta \varepsilon_{i j}\left(\vec{r}^{\prime}\right)\right\rangle$. Substituting Eq. (2) and Eq. (3) into Eq. (4) we obtain $\left\langle\delta \varepsilon_{i j}(\vec{r}) \delta \varepsilon_{i j}\left(\vec{r}^{\prime}\right)\right\rangle / C_{0}^{2}$

$$
\begin{aligned}
& x x: 10\left|\left\langle E_{x}^{(U V)} E_{x}^{\prime(U V) *}\right\rangle\right|^{2}+\left|\left\langle E_{y}^{(U V)} E_{y}^{\prime(U V) *}\right\rangle\right|^{2}+6\left|\left\langle E_{x}^{(U V)} E_{y}^{\prime(U V) *}\right\rangle\right|^{2}+2\left|\left\langle E_{y}^{(U V)} E_{z}^{\prime(U V) *}\right\rangle\right|^{2} \\
& y y: 2\left|\left\langle E_{x}^{(U V)} E_{x}^{\prime(U V) *}\right\rangle\right|^{2}+9\left|\left\langle E_{y}^{(U V)} E_{y}^{\prime(U V) *}\right\rangle\right|^{2}+6\left|\left\langle E_{x}^{(U V)} E_{y}^{\prime(U V) *}\right\rangle\right|^{2}+6\left|\left\langle E_{y}^{(U V)} E_{z}^{\prime(U V) *}\right\rangle\right|^{2} \\
& x y: \operatorname{Re}\left[\left\langle E_{x}^{(U V)} E_{x}^{\prime(U V) *}\right\rangle\left\langle E_{y}^{(U V)} E_{y}^{\prime(U V) *}\right\rangle+\left\langle E_{x}^{(U V)} E_{y}^{\prime(U V) *}\right\rangle^{2}+c . c .\right],
\end{aligned}
$$

where we use shorthand notations $E_{i}^{(U V)}(\vec{r}) \rightarrow E_{i}^{(U V)}$ and $E_{i}^{(U V)}\left(\vec{r}^{\prime}\right) \rightarrow E_{i}^{\prime(U V)}$ and chose to show only a $2 \times 2$ section $(x-y)$ of the entire tensor, which will be relevant to our further discussion. c.c. represents complex conjugate. By substituting the UV field correlators from Sec. 2.2 we obtain the desired result. The spatial correlations in the photo-induced fluctuations of the dielectric tensor are evaluated numerically and shown in Fig. 3. We observe that the general structure of the correlator is similar to that of $\left|\left\langle E_{x}^{(U V)} E_{x}^{\prime(U V) *}\right\rangle\right|^{2}$, even for $\left\langle\delta \varepsilon_{x y}(\vec{r}) \delta \varepsilon_{x y}\left(\vec{r}^{\prime}\right)\right\rangle$. The relative magnitudes of different components depend on such factors as the fabrication geometry, c.f. Fig. 1, which allows one to design disordered optical fibers with a desired set of statistical properties.

The natural scale to measure and compare magnitudes of different quantities in Eq. (10), Eq. (11) and Eq. (12) is $\Delta \varepsilon_{x x}$. We find that under the experimental conditions $\Delta \varepsilon_{y y} / \Delta \varepsilon_{x x} \simeq 0.7$, $\left\langle\delta \varepsilon_{x x}^{2}\right\rangle / \Delta \varepsilon_{x x}^{2} \simeq 0.5,\left\langle\delta \varepsilon_{y y}^{2}\right\rangle / \Delta \varepsilon_{x x}^{2} \simeq 0.16$, and $\left\langle\delta \varepsilon_{x y}^{2}\right\rangle / \Delta \varepsilon_{x x}^{2} \simeq 0.04$. These values will become important when we interpret the results of the experiment further below.

\section{Hybrid coupled-power / coupled-mode theory}

\subsection{Motivation}

Even (nominally) single-mode fiber (SMF) supports two propagating modes with orthogonal polarizations. Geometric and stress imperfections along the length of the single-mode fiber give rise to randomly varying birefringence [29]. Two perpendicular polarization states of the same mode become coupled with the characteristic length on the order of kilometers. This effect, known as polarization modal dispersion (PMD), can be described based on coupledmode equations formalism [30].

In MMF different modes generally propagate with different group delays, an effect known as inter-modal dispersion. We note that, although inter-modal dispersion in MMF causes the 
output intensity profile to change with the propagation distance, it does not lead to the mixing of modes as in PMD in SMF. Meanwhile, various imperfections in the fiber geometry do give rise to mode-coupling described in language of coupled-power equations $[2,18]$. Increasing concentrations of imperfections to stimulate coupling between the guided modes also inevitably results in coupling to the radiative modes.

In MMF with designed disorder considered in this work both cross- and intra-polarization couplings between co-propagating modes occur on the same very short scale of several centimeters. Meanwhile, the radiative leakage occurs at scale one order of magnitude longer. This relationship between different length scales will inform our further analysis.

In choosing the applicable theoretical approach for this problem we were guided by the following considerations. In our system, the coupled-power method cannot provide an adequate description of an evolution of the state of polarization (induced by birefringence) because it does not account for phases of the perpendicular polarization states of the same mode. On the other hand, the couple-mode approach becomes too cumbersome because we consider MMFs supporting a relatively large number (on the order of tens) of modes. This also disallows the continuum approximation in the coupled-power method [19] because this number is not sufficiently large.

\subsection{Separation into deterministic and stochastic contributions}

In Sec. 2.3 we obtained Eq. (10) and Eq. (11) for the statistical average dielectric tensor which shows that the fiber becomes (linearly) birefringent. Hence we begin our analysis by expressing the electric field in terms of $x$ - and $y$ - linearly polarized co-propagating modes (orientation of the axes is shown in Fig. 1) in the weakly guiding birefringent step-index fiber with the average dielectric tensor

$$
E_{i}(x, y, z) \approx \sum_{v} c_{v, i}(z) e^{i\left(\omega t-\beta_{v}\right) z} \mathscr{E}_{v, i}(x, y) .
$$

Here we adopt mode notations with two subscripts: $v$ enumerates $N / 2$ linearly polarized (LP) modes whereas $i=\{x, y\}$ explicitly denotes the polarization of the mode. $\beta_{v} \equiv\left(\beta_{v, x}+\beta_{v, y}\right) / 2$ is the propagation constant averaged over two polarization states of $v^{\prime}$ th mode. Birefringence results in mode dispersion $\Delta \beta_{v} \equiv\left(\beta_{v, x}-\beta_{v, y}\right)$. The transverse field profiles $\mathscr{E}_{V, i}(x, y)$ are assumed to be normalized as $\beta_{v, i} \iint\left[\mathscr{E}_{v, i}(x, y) \mathscr{E}_{v^{\prime}, i^{\prime}}(x, y)\right] d x d y=\delta_{v v^{\prime}} \delta_{i i^{\prime}}$, where $\delta_{v v^{\prime}}$ and $\delta_{i i^{\prime}}$ are the Kronecker symbols. In these notations $c_{v, i}(z)$ satisfy coupled-mode equations

$$
d c_{v, i}(z) / d z=\left( \pm i \Delta \beta_{v} / 2-\alpha / 2\right) c_{v, i}(z)+\sum_{v^{\prime}, i^{\prime}} \mathscr{K}_{v v^{\prime}, i^{\prime}}(z) c_{v^{\prime}, i^{\prime}}(z) e^{i\left(\beta_{v}-\beta_{v^{\prime}}\right) z}
$$

where $\alpha$ is the loss coefficient (assumed to be mode independent [31]) and $\mathscr{K}_{v v^{\prime}, i i^{\prime}}(z)=$ $\left(\omega^{2} / 2 i c^{2}\right) \iint \mathscr{E}_{V, i}(x, y) \delta \varepsilon_{i i^{\prime}}(x, y, z) \mathscr{E}_{V^{\prime}, i^{\prime}}(x, y) d x d y$ are mode coupling coefficients. The sign of the polarization dispersion term is chosen positive for $x$ and negative for $y$ modes. We note that $x-y$ axes depicted in Fig. 1 constitute principal axes of the statistically averaged dielectric tensor, c.f. Eq. (10) and Eq. (11).

Next we separate the modal coefficients into a deterministic (ballistic) and random components via

$$
c_{v, i}(z)=\left\langle c_{v, i}(z)\right\rangle+\delta c_{v, i}(z),
$$

where $\langle\ldots\rangle$ denotes disorder average. By definition $\left\langle\delta c_{v, i}(z)\right\rangle \equiv 0$. Below we describe the deterministic component with coupled-mode (amplitude) equations whereas the random contribution will be treated within the coupled-power approach. In doing so, we explicitly account for polarization rotation only in the transition regime (via coupled-mode equation for the amplitudes) of sufficiently short samples when the ballistic signal has not yet had a chance to 
reach the equilibrium mode distribution. As the energy is removed from the ballistic signal and transfered into randomly phased component, described by $\delta c_{v, i}(z)$, the effects of inter-mode scattering and polarization dispersion compete. Therefore, the coupled-power description of $\delta c_{v, i}(z)$ is justified because, as it will be evident from the experimental analysis in Sec. 4, both processes occur on the same characteristic length scale and because the inter-mode scattering also involves cross-polarization coupling on the similar scale, c.f. Eqs. (12).

\subsection{Coupled-mode description of mode amplitudes}

Obtaining the evolution equations for $\left\langle c_{v, i}(z)\right\rangle$ from Eq. (15) involves the task of computing $\left\langle\mathscr{K}_{v v^{\prime}, i^{\prime}}(z) c_{v^{\prime}, i^{\prime}}(z)\right\rangle$. The latter can be accomplished by employing approach used in an analysis of dynamical systems. First, we formally integrate Eq. (15) to obtain $c_{v^{\prime}, i^{\prime}}(z)$ on the left hand side of the equation. Then we multiply both sides by $\mathscr{K}_{\eta v, j i}(z)$ and perform a statistical average. Assuming that $\delta \varepsilon_{i j}(x, y, z)$ is a random delta-correlated Gaussian process, enables us to compute the average. This assumption is justified because there is a large disparity in correlation scales between $c_{v^{\prime}, i^{\prime}}(z)$ and $\delta \varepsilon_{i j}(x, y, z)$ - centimeters and $\lambda_{U V}$ respectively, c.f. Sec. 2.2. We obtain

$$
d\left\langle c_{v, i}(z)\right\rangle / d z=\left( \pm i \Delta \beta_{v} / 2-\alpha / 2-h_{v, i} / 2\right)\left\langle c_{v, i}(z)\right\rangle,
$$

with coupling coefficients are defined by

$$
\begin{aligned}
h_{v, i} & =\sum_{v^{\prime \prime}, i^{\prime \prime}} h_{v v^{\prime \prime}, i^{\prime \prime}} \\
h_{v v^{\prime \prime}, i i^{\prime \prime}} & =\left(\omega^{4} / 4 c^{4}\right)\left\langle\delta \varepsilon_{i i^{\prime \prime}} \delta \varepsilon_{i^{\prime \prime} i}\right\rangle V_{i i^{\prime \prime} i^{\prime \prime} i} \iint \mathscr{E}_{v, i}^{2}(x, y) \mathscr{E}_{v^{\prime \prime}, i^{\prime \prime}}^{2}(x, y) d x d y,
\end{aligned}
$$

where we approximated $\left\langle\delta \varepsilon_{i i^{\prime \prime}}(\vec{r}) \delta \varepsilon_{i^{\prime \prime} i}\left(\vec{r}^{\prime \prime}\right)\right\rangle \simeq\left\langle\delta \varepsilon_{i i^{\prime \prime}} \delta \varepsilon_{i^{\prime \prime} i}\right\rangle V_{i i^{\prime \prime} i^{\prime \prime} i} \delta\left(\vec{r}-\vec{r}^{\prime \prime}\right) . V_{i i^{\prime \prime} i^{\prime \prime} i}$ is the correlation volume of the UV speckle pattern of the fabricated disorder, c.f. Sec. 2.2 and Ref. [17]. In arriving at Eq. (16) we neglected the terms involving $\left\langle c_{v^{\prime}, i^{\prime}}(z)\right\rangle$ cross channel scattering, $v \neq v^{\prime}, i^{\prime} \neq i$, on the right hand side. This is justified because: (a) for co-polarized modes coupling term involves factor $\iint \mathscr{E}_{V, i}(x, y) \mathscr{E}_{v^{\prime \prime}, i^{\prime \prime}}^{2}(x, y) \mathscr{E}_{v^{\prime}, i}(x, y) d x d y$ which is small for $v \neq v^{\prime}$ due to orthogonality of $\mathscr{E}_{V, i}(x, y)$; (b) for cross-polarized modes the factor $\left\langle\delta \varepsilon_{i i^{\prime \prime}} \delta \varepsilon_{i^{\prime \prime} i^{\prime}}\right\rangle$ is small $\left(i \neq i^{\prime}\right)$, c.f. Sec. 2.3 .

We conclude this section by writing the solution of the derived Eq. (16)

$$
\left\langle c_{v, i}(z)\right\rangle \simeq c_{v, i}(0) \exp \left[\left( \pm i \Delta \beta_{v} / 2-\alpha / 2-h_{v, i} / 2\right) z\right]
$$

where $c_{v, i}(0)$ denote the modal amplitudes upon injection into the fiber at $z=0$. The solution above describes the evolution of the state of polarization of $v^{\prime}$ th mode and its exponential attenuation due to loss ( $\alpha$ term) and decoherence $\left(h_{v, i}\right.$ term). This latter process transfers power from the ballistic to random component, which we treat next.

\subsection{Coupled-power equations for random component}

Derivation for the coupled power equations [17] satisfied by $P_{v, i}^{(\delta)} \equiv\left\langle\left|\delta c_{v, i}\right|^{2}\right\rangle$ leads to a new source term $h_{v, i} P_{v, i}^{(b)}(z) \equiv h_{v, i}\left|\left\langle c_{v, i}(z)\right\rangle\right|^{2}$ in the otherwise cannonical coupled power equation [18]:

$$
d P_{v, i}^{(\delta)} / d z=h_{v, i} P_{v, i}^{(b)}-\alpha P_{v, i}^{(\delta)}+\sum_{v^{\prime}, i^{\prime}} h_{v v^{\prime}, i^{\prime}}\left(P_{v^{\prime}, i^{\prime}}^{(\delta)}-P_{v, i}^{(\delta)}\right) .
$$

The source term represents in-flux from the ballistic component described by Eq. (16). The above equation should be supplemented with the initial conditions $P_{v, i}^{(\delta)}(0)=0$.

\#160441 - \$15.00 USD

(C) 2012 OSA
Received 22 Dec 2011; revised 11 Jan 2012; accepted 11 Jan 2012; published 30 Jan 2012 13 February 2012 / Vol. 20, No. 4 / OPTICS EXPRESS 3628 
The approximate solution of Eq. (20) can be obtained at both small and large values of $z$. For modes with non-zero (ballistic) source terms we find $P_{v, i}^{(\delta)}(z) \approx h_{v, i}\left|c_{v, i}(0)\right|^{2} \times z$ and $P_{v, i}^{(\delta)}(z) \approx$ $\left[\sum_{v^{\prime}, i^{\prime}} h_{v v^{\prime}, i i^{\prime}} h_{v^{\prime}, i^{\prime}}\left|c_{v^{\prime}, i^{\prime}}(0)\right|^{2}\right] \times z^{2}$ for the rest. This solution is valid for $z<\min \left[h_{v, i}\right]$. For $z \gg$ $\sigma_{2}$ when the source terms become negligible, the power becomes equipartitioned among all $N$ modes $P_{v, i}^{(\delta)}(z) \approx(1 / N) \exp [-\alpha z]$. Here $\sigma_{2} \sim h_{v v^{\prime}, i i^{\prime}}$ denotes the second smallest eigenvalue of the secular equation $\operatorname{det}\left[h_{v v^{\prime}, i i^{\prime}}+\left(\sigma-h_{v, i}\right) \delta_{v v^{\prime}} \delta_{i i^{\prime}}\right]=0[17,18]$.
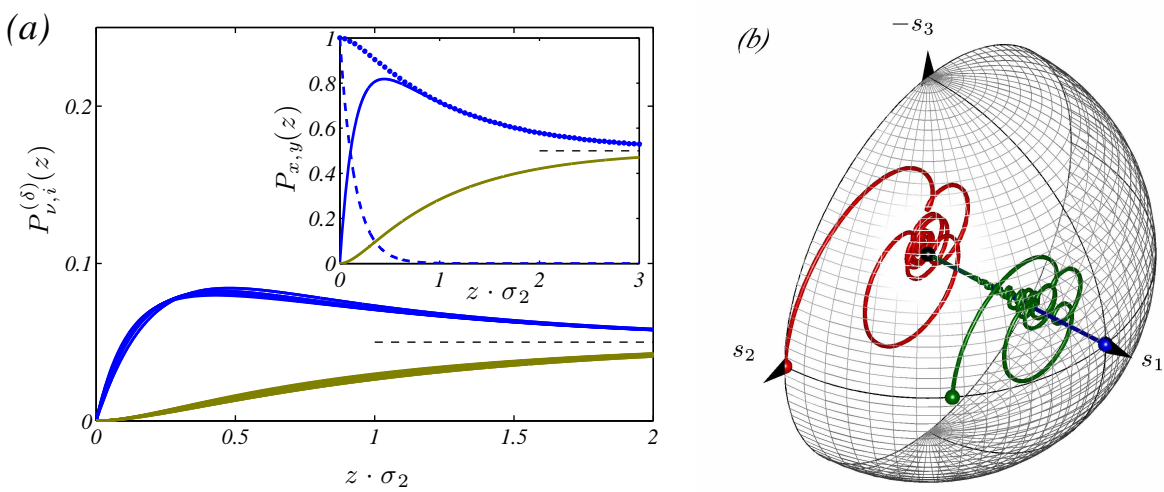

Fig. 4. (a) Runge-Kutta numerical solution of Eqs. (20) with $\alpha=0$. Only $x$-polarized LP modes are exited: $c_{V, x}(0)=(2 / N)^{1 / 2}$. The power becomes equally distributed among the modes of both polarizations ( $x$ - blue, $y$-gold) before equilibrating at $1 / N$ level shown as a dashed line. $\sum_{v} P_{v, i}^{(\delta)}(z)$ converging at $1 / 2$ are shown in the inset. The ballistic component $\sum_{v} P_{v, x}^{(b)}(z)$ and the total power in the $x$ polarization $\sum_{v} P_{v, x}^{(b)}(z)+\sum_{v} P_{v, x}^{(\delta)}(z)$ are depicted with dashed and dotted lines respectively. (b) Evolution along the fiber length of elements of the Stokes vector $\vec{s}(z)$. The Poincare sphere plot describes the transition from the linearly polarized light with the degree of polarization $\mathscr{P} \equiv|\vec{s}|=1$ at $z=0$ to unpolarized light with $\mathscr{P}=0$ in the limit $z \rightarrow \infty$. Blue, green and red lines correspond to the light linearly polarized at $\phi=0, \pi / 8$, and $\pi / 4$ with respect to the $x$ primary axis of the average dielectric tensor respectively.

To obtain solution for arbitrary $z$ we use the forth order Runge-Kutta method to solve Eqs. (20) numerically under experimentally relevant conditions; $\alpha=0$ is assumed for clarity. At $z=0$ the only non-zero terms are $c_{v, x}(0)=(2 / N)^{1 / 2}$. Figure $4\left(\right.$ a) depicts $P_{v, x}^{(\delta)}(z)$ and $P_{v, y}^{(\delta)}(z)$ as blue and gold lines respectively. $P_{v, x}^{(\delta)}(z)$ are peaked at small $z$ before equilibration is reached among the modes of both polarizations. The dashed line depicts the $1 / N$ level. We observe that efficient cross-polarization coupling due to induced birefringence makes intraand inter-polarization equilibration occur at similar lengths scales, i.e. $\sigma_{2}^{-1} \sim 2 \sigma_{3}^{-1}$ which is explicitly confirmed numerically. The inset in Fig. 4(a) depicts $\sum_{v} P_{v, i}^{(\delta)}(z)$ demonstrating an equilibration at $1 / 2$ level shown as a black dashed line. Also depicted are the ballistic component $\sum_{v} P_{v, x}^{(b)}(z)$, dashed line, and the total power in $x$ polarization $\sum_{v} P_{v, x}^{(b)}(z)+\sum_{v} P_{v, x}^{(\delta)}(z)$, dotted line.

\subsection{De-polarization and Stokes parameters}

The direct consequence of the cross-polarization mode coupling caused by the induced birefringence, c.f. Eq. (12), is a de-polarization of the initial excitation. This process is conveniently described by Stokes parameters [32]. Separation of the modal amplitudes into the deterministic 
(ballistic) and random contributions, c.f. Eq. (15), enables us to obtain the following expressions:

$$
\begin{array}{ll}
S_{0}=\sum_{v=1}^{N / 2}\left(P_{v, x}^{(b)}+P_{v, x}^{(\delta)}-P_{v, y}^{(b)}-P_{v, y}^{(\delta)}\right) ; & S_{1}=\sum_{v=1}^{N / 2}\left(P_{v, x}^{(b)}+P_{v, x}^{(\delta)}-P_{v, y}^{(b)}-P_{v, y}^{(\delta)}\right) ; \\
S_{2}=\sum_{v=1}^{N / 2}\left(\left\langle c_{v, x}\right\rangle\left\langle c_{v, y}^{*}\right\rangle+\left\langle c_{v, x}^{*}\right\rangle\left\langle c_{v, y}\right\rangle\right) ; & S_{3}=i \sum_{v=1}^{N / 2}\left(\left\langle c_{v, x}\right\rangle\left\langle c_{v, y}^{*}\right\rangle-\left\langle c_{v, x}^{*}\right\rangle\left\langle c_{v, y}\right\rangle\right) .
\end{array}
$$

Three-component Stokes vector is defined as $s_{1-3}=S_{1-3} / S_{0}$. In these notations the fully polarized light, regardless of its state of polarization, corresponds to the Stokes vector at the surface of the Poincaré sphere $|\vec{s}|=1$. In contrast, a completely unpolarized light has the state of polarization $\mathscr{P} \equiv|\vec{s}|=0$.

Figure 4 depicts the evolution of the Stokes vector along the length of the fiber. Blue, green and red lines correspond to the initial linear polarizations at the angles $\phi=0, \pi / 8, \pi / 4$ with respect to the principal axis of the fiber: $c_{v, x}=(2 / N)^{1 / 2} \cos (\phi), c_{v, y}=(2 / N)^{1 / 2} \sin (\phi)$ is assumed. In each case, we observe a relaxation of the state of polarization from $\mathscr{P}=1$ at $z=0$ to $\mathscr{P}=0$ at $z \gg \sigma_{2}^{-1}$. This concludes our demonstration of complete mixing on the same short length scale $\sigma_{2}^{-1}$ of the both co- and cross-polarized modes in the artificially disordered fiber.

\section{Experimental corroboration}

\subsection{Experimental setup}

In this experiment we employed a step-index silica fiber doped by Ge (PS1250/1500 of Fibercore). The main parameters of the fiber are a core diameter of $7.66 \mu \mathrm{m}$, cladding diameter of $125 \mu \mathrm{m}$, and numerical aperture (NA) of 0.13 , with the refractive indices of the core and the cladding being 1.463 and 1.457 , respectively. Three samples of fiber (about $20 \mathrm{~cm}$ ) included the disordered part of 2, 4 and $12 \mathrm{~cm}$ respectively. Fabrication and characterization of the volume disorder is described in details in Ref. [17].

At the input of the optical fiber the polarized light goes through a half wave-plate and a linear polarizer oriented along axis $x^{\prime}$, which makes angle $\phi$ with respect to the principal axis of the fiber $x$. The output light was detected separately for both polarizations: a) after passing through a polarizer of the same orientation as at the input $\left(x^{\prime} x^{\prime}\right.$ - polarization), or b) perpendicularly polarized ( $x^{\prime} y^{\prime}$ - polarization). We analyzed the output light of each polarization independently. Measurement was repeated 10 times for different random bending of the fiber. The bending of the fiber was sufficient to change the disorder but not sufficient to introduce appreciable additional birefringence. Both polarizers were rotated synchronously by $10^{\circ}$ (i.e. $\phi$ was incrementally increased) and measurements were repeated.

At the probe laser light wavelength $\lambda=543 \mathrm{~nm}$ used in measurements, the number of guided LP modes (counting both polarizations) is $N=20$. The average integrated intensity of outgoing light for two perpendicular orientations of the polarizer are presented in Fig. 5 for rotations in the interval $0^{\circ}-90^{\circ}$.

\subsection{Comparison of theory and polarization-resolved measurement}

After obtaining the solutions for $\left\langle c_{v, i}(z)\right\rangle$ and $P_{v, i}^{(\delta)}(z)$ describing the evolution of the ballistic and stochastic components in Eq. (15) in the principal axes coordinate system $x-y$, we perform the coordinate transformation to the axes $x^{\prime}-y^{\prime}$ rotated by angle $\phi$. We obtain

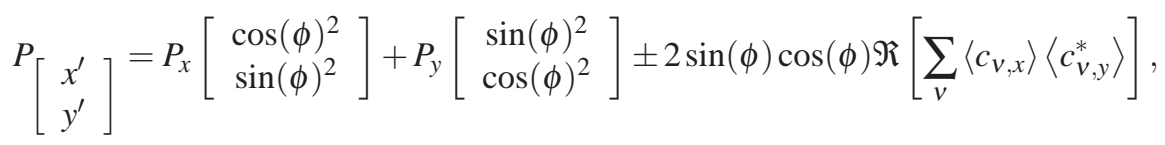

\#160441 - \$15.00 USD

(C) 2012 OSA
Received 22 Dec 2011; revised 11 Jan 2012; accepted 11 Jan 2012; published 30 Jan 2012 13 February 2012 / Vol. 20, No. 4 / OPTICS EXPRESS 3630 
where $P_{i} \equiv\left|\left\langle c_{v, i}\right\rangle\right|^{2}+P_{v, i}^{(\delta)}$ and $\Re[\ldots]$ denotes the real part.

Eq. (22) can be evaluated numerically as in Sec. 3.4. However, it is illuminating to find a closed-form analytical expression describing the evolution of power in each polarization $P_{i^{\prime}}$. This task can be accomplished with an assumption that the asymptotic expression for $P_{i}(z)=$ $\left[\left(P_{i}(0)-1 / 2\right) \exp \left(-\sigma_{2} z\right)+1 / 2\right] \exp (-\alpha z)$ is valid for the entire range of $0<z<\infty$. The inset in Fig. 4 shows the adequacy of such an approximation. We arrive at our final result

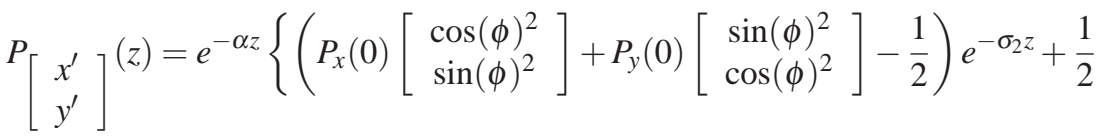

$$
\begin{aligned}
& \left. \pm 2 \sin (\phi) \cos (\phi) \frac{2}{N} \sum_{v} P_{v, x}^{1 / 2}(0) P_{v, y}^{1 / 2}(0) \exp \left(-\frac{h_{v, x}+h_{v, y}}{2} z\right) \cos \left(\Delta \beta_{v} z\right)\right\},
\end{aligned}
$$

where, as previously, we take $P_{x}(0)+P_{y}(0)=1$. The above expression describes the following effects: (i) attenuation due to radiative losses $-\alpha$ term; (ii) assymptotic equal distribution of power over all $N$ co-propogating modes of the fiber $-\sigma_{2}$ term; (iii) de-polariation of the incindent light $-h_{v, i}$ term; (iv) change of the polarization state is the decaying ballistic signal $-\Delta \beta_{v}$ term. Furthermore, the expression above conserves power at every $z$. Figure 5 demonstrates that Eq. (23) describes the experiment well: it captures both the polarization and length dependences of the transmitted signal.
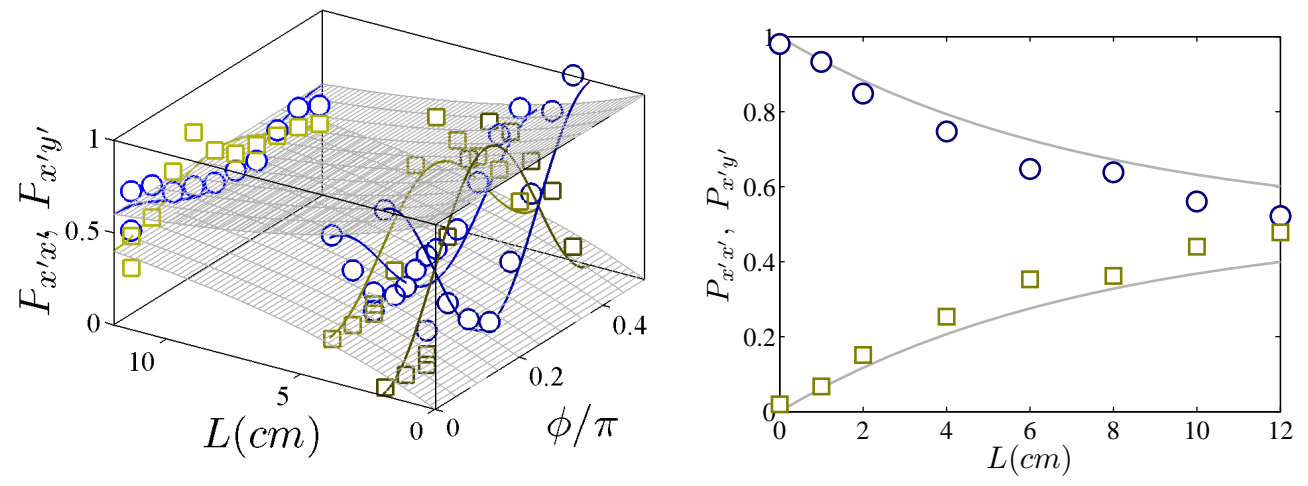

Fig. 5. (a) Experimental data in $L=2,4$ and $12 \mathrm{~cm}$ samples for the ensemble-averaged transmission for $x^{\prime} x^{\prime}$ (circles) and $x^{\prime} y^{\prime}$ (squares) polarization channels as the function of the angle $\phi$ between $x^{\prime}$ and the principal axis $x$. In all cases the incident light is polarized along $x^{\prime}$ axis. For clarity, the data is normalized as $P_{x^{\prime} x^{\prime}}(z) \rightarrow P_{x^{\prime} x^{\prime}}(z) / \int\left(P_{x^{\prime} x^{\prime}}(z)+P_{x^{\prime} y^{\prime}}(z)\right) d \phi$ and similarly for $P_{x^{\prime} y^{\prime}}(z)$ to eliminate the effect of attenuation $\times \exp (-\alpha z)$. Solid lines show the theoretical fit with Eq. (23) by combining the $\sum_{V}$ term into a single fitting parameter. Two converging surface envelopes $1 / 2 \pm \exp \left(-\sigma_{2} z\right) / 2$ are shown a guide for an eye demostrating almost complete mode mixing at $L=12 \mathrm{~cm}$. (b) Normalized by $P_{x}(z)+P_{y}(z)$ to eliminate the $\exp (-\alpha z)$ factor, $P_{x}(z), P_{y}(z)$ show convergence toward $1 / 2$. To acheive alighnment with the principal axes of fiber, the experimental data (symbols) was obtained in-situ during fabrication of the additional segments of disordered fiber. Solid lines obtained for $\phi=0$ from the fit in (a) show somewhat slower decay, that is attributed to unintentional twisting in process of generating ensemble realizations by bending of the fiber.

\#160441 - \$15.00 USD

(C) 2012 OSA
Received 22 Dec 2011; revised 11 Jan 2012; accepted 11 Jan 2012; published 30 Jan 2012 13 February 2012 / Vol. 20, No. 4 / OPTICS EXPRESS 3631 


\section{Conclusion}

In this work we obtained two main results. First, beginning with a microscopical model of photo-sensitivity in a germano-silicate glasses, we analytically derived formulae describing the spatial correlations between the components of the dielectric tensor, c.f. Sec. 2.3. We made the connection between the statistical properties, including the polarization statistics, of the incident UV light and those of the artificial disorder inscribed in the core of the fiber. Importantly, as it has been shown by Korotkova in Ref. [33], the statistics of (the UV) light changes when the light propagates in a free space. This suggests that the spatial properties of the UV light, and hence of the artificial disorder, can be manipulated by altering the illuminations geometry during the disorder writing process.

The second result concerns the description of the light propagation in the fiber with the artificially correlated disorder. Our previous study based on coupled power theory [17] could not explain strong mixing of cross-polarized co-propagating modes observed experimentally. We suggested that an induced birefringence might be the cause. This work shows that the actual answer is more complicated. On one hand, the existence of the linear birefringence on average, c.f. Eq. (10) and Eq. (11), causes only a periodic evolution of the two polarization states of each mode in the fiber. By itself this effect does not lead to equilibration of power among all modes in two polarization channels. Instead, the coupling is the result of the non-zero correlations between the off-diagonal components of the dielectric tensor, c.f. Eq. (12), which in turn are caused by the non-trivial correlations between $x$ - and $y$ - components of the UV writing beam, c.f. Fig. 2. Unlike intra-polarization coupling, the magnitude of the cross-polarization coupling is directly related to existence of the longitudinal component of the UV field used in fabrication of disorder. Thus, our choice of the near-field illumination (small reparation between diffuser and fiber in Fig. 1) is critical. Our theory predicts significantly weaker coupling between orthogonally-polarized modes in the paraxial regime when the only remaining coupling due to bending-stresses may become dominant.

Modeling light propagation in the disordered birefringent fiber poses a challenge for a coupled-power description. In fact, it becomes inadequate because coherent process of evolution of polarization cannot be captured. To overcome this limitation, in this work we developed a hybrid theory which treats the deterministic (ballistic) part of the light via coupled-amplitude equations, whereas the randomly-phased component is treated with a coupled-power equations, c.f. Sec. 3. Neglecting the polarization change in the random component is justified in our artificially disordered fiber because the process of scattering occurs on a very short length scale on the order of $1 \mathrm{~cm}$.

Comparison with experiment in Sec. 4 suggests that very short $\sim 10 \mathrm{~cm}$ segments of the fabricated multi-mode fibers with designed disorder can be used as mode-scrambler / descrambler mixing efficiently all modes with both states of polarization. Furthermore, we previously estimated and confirmed experimentally that the mixing can be accomplished without significant radiative losses, $3.6 d B$ in Ref. [17]. This makes our fibers with custom-made disorder suitable for such applications as e.g. hardware encryption and power-management in high-power fiber laser systems. The complete mode mixing is also required to achieve the maximum channel capacity in MIMO transmission in the multi-mode optical fibers.

\section{Acknowledgments}

The work at Missouri S\&T was supported by the University of Missouri Research Board and by the National Science Foundation Grant No. DMR-0704981. NPP and EIC would like to acknowledge a support by CONACYT (México), under grant UCM-42127. 